\title{
LINEARISED EVAPORATION ABOUT A SHALLOW HALF-PLANE POND
}

\author{
G. J. WEIR ${ }^{1}$
}

(Received 13 May 1991; revised 21 November 1991)

\begin{abstract}
The Wiener-Hopf technique is applied to the quasi-linear infiltration problem of flow from a shallow half-plane pond. Fully-saturated conditions hold immediately under the pond, while on the surface away from the pond the linearised evaporative loss is assumed to be proportional to the local relative permeability.

Evaporation from the non-wetted region increases the water flow from the pond into the soil, thereby coupling to the effects from capillarity. Linearised evaporation introduces an additional length scale and additional logarithmic expressions to those derived previously. The total rate of volumetric flow into the soil from the pond per unit length of perimeter, in addition to the usual gravity flow, increases somewhat slowly as evaporation increases. The most extreme case considered in this paper yielded an additional flow rate $63 \%$ greater than that obtained in the absence of evaporation.

The interaction between evaporation and capillarity is enhanced in poorlydraining soils, where the reduced ability to transmit liquid water need not be compensated by a corresponding reduction in evaporative losses. However, in freelydraining soils the interaction between evaporation and capillarity is probably small.
\end{abstract}

\section{Introduction}

The interaction between gravity, capillarity and geometry has been the subject of recent theoretical studies in Australia by $R$. T. Waechter and J. R. Philip [7], and in New Zealand by G. J. Weir [8]. This paper's aim is to modify a previous approach so that interactions from evaporation can also be discussed.

\footnotetext{
${ }^{1}$ Appl. Math. Group, Dept. of Scientific and Industrial Res., P.O. Box 1335, Wellington, N.Z. (C)Copyright Australian Mathematical Society 1992, Serial-fee code 0334-2700/92
} 
Evaporation from unsaturated soils can be classified (Hillel [2]) as being either (almost) isothermal or non-isothermal. Both types of evaporation involve highly nonlinear processes, especially because of simultaneous liquid and vapour processes, and thermal effects. Further, evaporation rates depend on multiple parameters, such as wind speed, energy fluxes, water table depths, etc.

Some non-evaporative problems, by contrast, can be satisfactorily simplified and nondimensionalised to yield linear equations with no free parameters. This paper considers such a problem, except that one boundary condition is modified in a linear manner to allow an evaporative loss from non-wetted regions. This idealised model problem allows significant progress to be made analysing the corresponding linear equations.

In contrast, a discussion of the full physics of evaporative processes in soil leads to very difficult equations. The basic equations (Pruess [6]) describe conservation of water, air and energy in a porous medium. Water and air occur in both the liquid and vapour phases, with Henry's law approximating the ratio of mass fractions of each component in each phase. Darcy's law, together with relative permeability functions, will describe the flows of each phase. Since capillarity causes each phase to be at different pressures, evaporative processes in porous media are then described by three nonlinear second-order diffusive equations.

Mathematical progress is made disregarding the vapour phase, and the energy equation. This results in a single second-order (Buckingham's) equation approximating flow of liquid water in soil. The medium is therefore assumed isothermal, and air is assumed to be at atmospheric pressure and have negligible density.

However, the vapour phase cannot always be ignored. In regions of very low liquid relative permeability, the main mode of transport can occur in the vapour phase. This difficulty is disregarded by arguing that in such regions the mass flows are small, and therefore ignorable, relative to flows in regions of high liquid relative permeability where the dominant mode of transport occurs in the liquid phase.

Any discussion of evaporation necessarily involves the vapour phase, yet inclusion of vapour transport introduces apparently intractable mathematical difficulties. This paper attempts to avoid this difficulty by allowing a loss of water from the soil to the atmosphere, which therefore approximates, crudely at least, an evaporative loss. Additionally, in order to proceed mathematically, we ignore the vapour phase in the soil, which implies that our analysis should be valid in regions of high liquid relative permeability, but invalid (together with many other published results) in regions where vapour transport dominates. 
The ability of the air to transport water vapour from soil is often much greater than the ability of the soil to supply water to the surface. In such cases, liquid flow processes within the soil may be rate-determining, which may justify their study, even when coupled with evaporative boundary conditions. We shall proceed from this viewpoint, especially as it leads to interesting and suggestive mathematics.

\section{Model formulation}

A shallow half-plane overlies a homogeneous half-space of porous material (see Figure 1). The quasi-linear equation (Philip [4], Weir [8]) for unsaturated steady liquid flow in the porous medium is

$$
\nabla^{2} \kappa=\alpha \frac{\partial \kappa}{\partial Z}
$$

where $\nabla^{2}$ is the Laplacian in Cartesian coordinates $(X, Y, Z), \kappa$ relative permeability, $\alpha$ the usual constant quasi-linear inverse length parameter and $Z$ is directed vertically downwards. Coordinates are chosen so that the surface of the porous medium is $Z=0$, while the pond occupies the half-plane $Z=0, X<0$ (see Figure 1).

$$
\text { evaporation }
$$

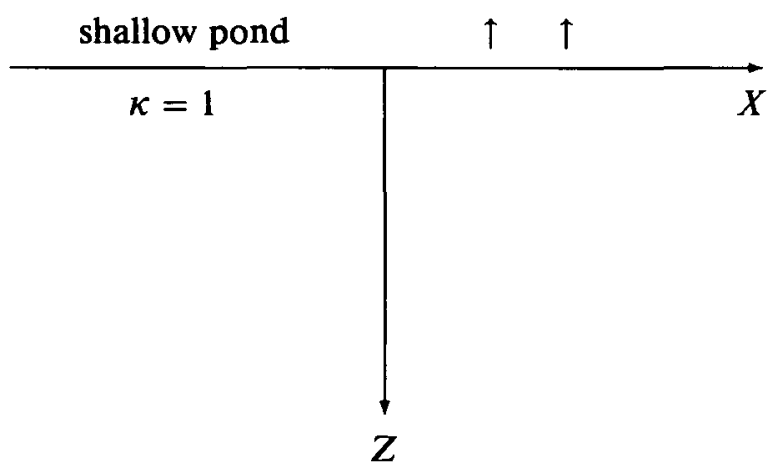

FIGURE 1. Schematic diagram of coordinate system for shallow half-plane pond infiltration problem.

The vertical volumetric flux $F_{Z}$ of fluid into the porous medium is

$$
F_{Z}=\frac{\rho g k_{1}}{\alpha \mu}\left(\alpha \kappa-\frac{\partial \kappa}{\partial Z}\right),
$$


where $g$ is gravitational acceleration, $k_{1}$ fully saturated permeability, and $\rho, \mu$ are the density and dynamic viscosity of water.

We shall assume for mathematical convenience that over non-wetted surface regions of the porous material, an evaporative loss produces a volumetric flux $F_{e}$ out of the porous material of magnitude

$$
F_{e}=\rho g k_{1} h \kappa / \alpha \mu, \quad Z=0,
$$

where $h$ is assumed a non-negative constant. Equation (3) represents a highly idealised model of evaporation, so chosen to produce a linear evaporative boundary condition. The parameter $h$ can be chosen so that the total linear evaporative loss has the required magnitude, but then the dependence of $F_{e}$ on distance from the pond will be physically questionable. Nevertheless, (3) does produce a surface mass loss, and so "linear" evaporation will be coupled to gravity and capillarity.

The coordinate transformation

$$
(x, y, z)=(\alpha / 2)(X, Y, Z),
$$

allows (1) to be nondimensionalised to

$$
\frac{\partial^{2} \kappa}{\partial x^{2}}+\frac{\partial^{2} \kappa}{\partial z^{2}}=2 \frac{\partial \kappa}{\partial z},
$$

since the problem is independent of $y$. Fully saturated conditions are assumed immediately under the pond,

$$
\kappa=1, \quad z=0, \quad x>0,
$$

and from (2) and (3),

$$
\frac{\partial \kappa}{\partial z}=2(1+h / \alpha) \kappa, \quad z=0, \quad x>0,
$$

since $F_{z}$ and $F_{e}$ are directed oppositely.

On physical grounds we may assume that $\kappa$ is continuous at the origin $(0,0)$, and then the solution of (5)-(7) may be represented as the Fourier transform,

$$
\kappa=\frac{i}{2 \pi} \int_{-\infty}^{\infty} \frac{G_{-}(0)}{\xi G_{-}(\xi)} \exp \left[i \xi x+z\left(1-\sqrt{1+\xi^{2}}\right)\right] d \xi,
$$

where the contour is indented above the origin in the $\xi$ plane.

Equation (5) is satisfied, as is (6), and (7) will also hold whenever

$$
G_{+}(\xi) G_{-}(\xi)=\gamma+\sqrt{1+\xi^{2}},
$$




$$
\gamma=1+2 h / \alpha
$$

and $G_{+}(\xi)$ is analytic above $-i$, and $G_{-}(\xi)$ analytic below $i$.

\section{Wiener-Hopf factorisation}

The factorisation required in (9) is obtained by defining

$$
G_{+}(\xi)=(\xi+i)^{\frac{1}{2}} H_{+}(\xi), \quad G_{-}(\xi)=(\xi-i)^{\frac{1}{2}} H_{-}(\xi),
$$

from which

$$
H_{+}(\xi) H_{-}(\xi)=1+\gamma(1+\xi)^{-\frac{1}{2}} .
$$

The factorisation of $H$ can then be performed directly (Carrier et al. [1], page 382). Alternatively, it can be shown that

$$
\begin{array}{r}
\frac{d}{d \xi} \ln \left[\frac{G_{-}(\xi)}{\sqrt{\gamma+\sqrt{1+\xi^{2}}}}\right] \\
=\frac{i}{2 \pi\left[1+\xi^{2}-\gamma^{2}\right]}\left[\sqrt{\gamma^{2}-1} \ln \left[\frac{\gamma-\sqrt{\gamma^{2}-1}}{\gamma+\sqrt{\gamma^{2}-1}}\right]\right. \\
\left.\quad-\frac{\xi \gamma}{\sqrt{1+\xi^{2}}} \ln \left[\frac{\sqrt{1+\xi^{2}}-\xi}{\sqrt{1+\xi^{2}}+\xi}\right]\right],
\end{array}
$$

which, apart from notational changes, is given in (b), page 91 of Noble [3].

The solution of (13) can then be written in the form

$$
\begin{gathered}
G_{-}(\xi) / G_{-}(0)=\Lambda \exp (i \theta), \\
\Lambda=\left[\left(\gamma+\sqrt{1+\xi^{2}}\right) /(1+\gamma)\right]^{\frac{1}{2}},
\end{gathered}
$$

and the definition of $\theta$ involves integrating the right-hand side of (14) between 0 and $\xi$. (The definition for $\theta$ here for zero $h$ corresponds to $-\theta$ in an earlier paper (Weir [8]).) $\Lambda$ and $\theta$ are respectively even and odd in $\xi$. All of the factors in the Fourier transform for $\kappa$ in (8) have now been derived.

From (9), $G_{-}=O\left(\xi^{\frac{1}{2}}\right)$ as $\xi \rightarrow \infty$. The integrand in (8), for $z=0$, is then $O\left(\xi^{3 / 2}\right)$ and so $\kappa$ is continuous at the origin. Similarly, $\partial \kappa / \partial z$ has an 
order $x^{-\frac{1}{2}}$ singularity on the surface $z=0$, near the origin. This singularity is physically acceptable, since it is shown in Section 5 to produce a finite flow from the pond.

\section{Asymptotics}

The qualitative behaviour of the solution above can be inferred intuitively. Immediately under the pond, conditions are fully saturated $(\kappa=1)$, and so sufficiently far to the left an almost saturated zone will occur beneath the pond. Similarly, sufficiently far to the right, $\kappa$ will be almost zero, and these two regions (where $\kappa$ is approximately unity or zero) will be joined by a boundary layer formed by capillarity and perhaps extending beneath the origin.

Quantitative expressions for the asymptotic behaviour of this boundary layer follow from

$$
\begin{gathered}
\kappa=\frac{1}{2}-\frac{1}{\pi} \int_{0}^{\infty} \frac{d \xi \sin (\xi x-\theta) \exp \left[z\left(1-\sqrt{1+\xi^{2}}\right)\right]}{\xi \Lambda}, \\
\sim \frac{1}{2} \operatorname{erfc}(x / \sqrt{2 z})+\frac{\theta^{\prime}(0) \exp \left(-x^{2} / 2 z\right)}{\sqrt{2 \pi z}}, \\
\theta^{\prime}(0)=\frac{\ln \left(\gamma+\sqrt{\gamma^{2}-1}\right)}{\pi \sqrt{\gamma^{2}-1}} .
\end{gathered}
$$

Selecting the contour in (8) as the real axis, except for an upwards indentation over the origin to avoid the pole, and using (14), gives (17). Keeping $x$ finite, but allowing $z$ to be large in (17) yields (18), while (19) follows from (13) and (14), being the derivative of $\theta$ at zero $\xi$.

For sufficiently large values of $z$, the asymptotic result in (18) shows that contours of constant $\kappa$ are parabolas of constant $x^{2} / 2 z$. This is well known [7] and so the new result is the correction term involving $\theta^{\prime}(0)$ in (18). Table 1 shows that increased evaporation tends to increase asymptotic symmetry, since the asymmetric term involving $\theta^{\prime}(0)$ in (18) decreases with increasing $\gamma$. This seems reasonable since, as $\gamma$ increases, $\kappa$ should tend to zero on the surface to the right, and this limiting solution satisfies the symmetry relationship [7],

$$
\kappa(x, z)+\kappa(-x, z)=1
$$


The asymptotic result in (18) was obtained by keeping $x$ finite, and allowing $z$ to be large Consequently (18) does not hold for large positive $x$ and sufficiently close to the surface $z=0$. In this region, the method of steepest descent can be applied to give (equation (13) in [4]),

$$
\kappa \sim \kappa^{*}(x, z) \exp \left(z-\sqrt{x^{2}+z^{2}}\right), \quad x>0,
$$

where $\kappa^{*}$ is a relatively slowly varying function of $x$ and $z$. For large $z$ and finite $x,(20)$ shows that the parabolic combination $x^{2} / 2 z$ will be important, which is consistent with (18), but for finite $z$ and large positive $x, \kappa$ decreases essentially as $\exp (-x)$.

An alternative derivation multiplies the integrand in $(8)$ by $G_{+}(\xi) / G_{+}(\xi)$ and deforms the contour about $i$ to $i \infty$, giving

$$
\begin{aligned}
& \kappa=G_{-}(0) \exp (z) \int_{1}^{\infty} G_{+}(i \xi) \exp (-\xi x) \Omega d \xi / \pi \xi, \\
& \mathbf{\Omega}=\left[\gamma \sin \left(z \sqrt{\xi^{2}-1}\right)+\sqrt{\xi^{2}-1} \cos \left(z \sqrt{\xi^{2}-1}\right)\right] /\left(\gamma^{2}+\xi^{2}-1\right),
\end{aligned}
$$

and so for small $z$ and large $x$,

$$
\kappa \sim \omega \int_{1}^{\infty} \sqrt{\xi^{2}-1} \exp (-\xi x) d \xi=\omega K_{1}(x) / x,
$$

where

$$
\omega=G_{-}(0) G_{+}(i)(1+\gamma z) \exp (z) /\left(\pi \gamma^{2}\right),
$$

and $K_{1}$ is the modified Bessel function of index one.

\section{Integral properties}

The total flow from the pond per unit boundary length and the total evaporative flow per unit boundary length are perhaps the quantities of primary interest in this paper. We show in this section that these can be determined exactly.

The total flow per unit length of boundary from the pond into the porous medium consists of a contribution from gravity, and additional flows $T F_{e}$ and $T F_{c e}$ induced respectively by evaporation and a combination of capillarity and evaporation. The total gravity contribution is infinite, being proportional to the wetted surface area. We shall ignore this gravity flow, and concentrate instead on the flows induced by evaporation and capillarity. This 
can be justified intuitively, since the surface effects from capillarity and evaporation will be restricted to a boundary layer about the pond's edge, whereas all realistic ponds have finite area.

We define, from (3) and (4),

$$
T F_{e}=\int_{0}^{\infty} F_{e} d X=\frac{2 \rho g k_{1} h}{\mu \alpha^{2}} \int_{0}^{\infty} \kappa d x, \quad \text { on } z=0,
$$

and from (2) and (4),

$$
T F_{c e}+T F_{e}=\frac{-\rho g k_{1}}{\alpha \mu} \int_{-\infty}^{0} \frac{\partial \kappa}{\partial z} d x, \quad \text { on } z=0 .
$$

The integral in (22) can be evaluated exactly since on $z=0$ from (8)-(10) and (14),

$$
\begin{aligned}
-\left.\int_{-\infty}^{0} \frac{\partial \kappa}{\partial z}\right|_{0} d x & =\frac{1}{2 \pi} \int_{-\infty}^{\infty} \frac{G_{-}(0)\left[\sqrt{1+\xi^{2}}+\gamma-\gamma-1\right] d \xi}{\xi^{2} G_{-}(\xi)} \\
& =\frac{1}{2 \pi} \int_{-\infty}^{\infty} \frac{G_{-}(0) G_{+}(\xi) d \xi}{\xi^{2}}-\frac{(\gamma+1)}{2 \pi} \int_{-\infty}^{\infty} \frac{G_{-}(0) d \xi}{\xi^{2} G_{-}(\xi)} \\
& =\frac{(1+\gamma)}{2 \pi} \oint \frac{G_{-}(0) d \xi}{\xi^{2} G_{-}(\xi)} \\
& =(1+\gamma) \theta^{\prime}(0) .
\end{aligned}
$$

Similarly, on $z=0$, from (8) and (23),

$$
\left.\int_{0}^{\infty} \kappa\right|_{0} d x=-\frac{1}{2 \pi} \int_{-\infty}^{\infty} \frac{G_{-}(0) d \xi}{\xi^{2} G_{-}(\xi)}=\theta^{\prime}(0) .
$$

Consequently, from (21) and (24),

$$
T F_{e}=2 \rho g k_{1} h \theta^{\prime}(0) / \mu \alpha^{2},
$$

while from (21), (22), (25) and (10),

$$
T F_{c e}=2 \rho g k_{1} \theta^{\prime}(0) / \alpha \mu .
$$

Equations (25) and (26) have dimensions length ${ }^{2} /$ time, and represent the total volumetric flows per unit time per unit boundary length for evaporation and capillarity-evaporation. Their ratio is simply

$$
T F_{e} / T F_{c e}=h / \alpha \text {. }
$$

The functional behaviour of (23)-(26) on $\gamma$ is tabulated in Table 1. 
TABLE 1. Functional dependence on $\gamma$ of nondimensional boundary flows induced by evaporation and capillarity.

\begin{tabular}{|r|c|c|c|}
\hline$\gamma$ & $\begin{array}{c}\text { capillarity- } \\
\text { evaporation } \\
\pi \theta^{\prime}(0)\end{array}$ & $\begin{array}{c}\text { additional } \\
\text { evaporation } \\
(\gamma-1) \pi \theta^{\prime}(0) / 2\end{array}$ & $\begin{array}{c}\text { total outflow } \\
(1+\gamma) \pi \theta^{\prime}(0) / 2\end{array}$ \\
\hline 1.0 & 1.000 & 0.000 & 1.000 \\
1.1 & 0.968 & 0.048 & 1.016 \\
1.2 & 0.938 & 0.094 & 1.032 \\
1.3 & 0.911 & 0.137 & 1.047 \\
1.5 & 0.861 & 0.215 & 1.076 \\
2.0 & 0.760 & 0.380 & 1.141 \\
3.0 & 0.623 & 0.623 & 1.246 \\
4.0 & 0.533 & 0.799 & 1.332 \\
5.0 & 0.468 & 0.936 & 1.404 \\
10.0 & 0.301 & 1.354 & 1.655 \\
\hline
\end{tabular}

Table 1 is constructed with the third column equalling the sum of the first two columns. Column 1 indicates that the capillarity-evaporation flow term decreases with increasing evaporation, whereas the additional total flow (not including the gravity flow) from the pond increases only slowly with increasing evaporation. For low evaporation rates the additional total flow results essentially from capillarity, whereas for large evaporation rates the additional total flow results essentially from evaporation. The evaporation and capillarity-evaporation flows are equal when $\gamma$ equals three.

\section{Estimation of $\gamma$}

This section presents a number of arguments which allow the magnitude of $\gamma$ to be estimated so that the total evaporative flow from the non-wetted region will approximate the value expected to occur in practice. We begin by presenting a qualitative picture of conditions about the pond's edge for almost isothermal evaporation.

In the non-wetted region, sufficiently far from the pond's edge, the relative permeability for liquid transport will decrease sufficiently so that vapour transport becomes important. This is sketched in Figure 2.

Vapour transport requires (in almost isothermal conditions) a gradient in relative humidity to maintain the diffusive flow of water vapour. But in 


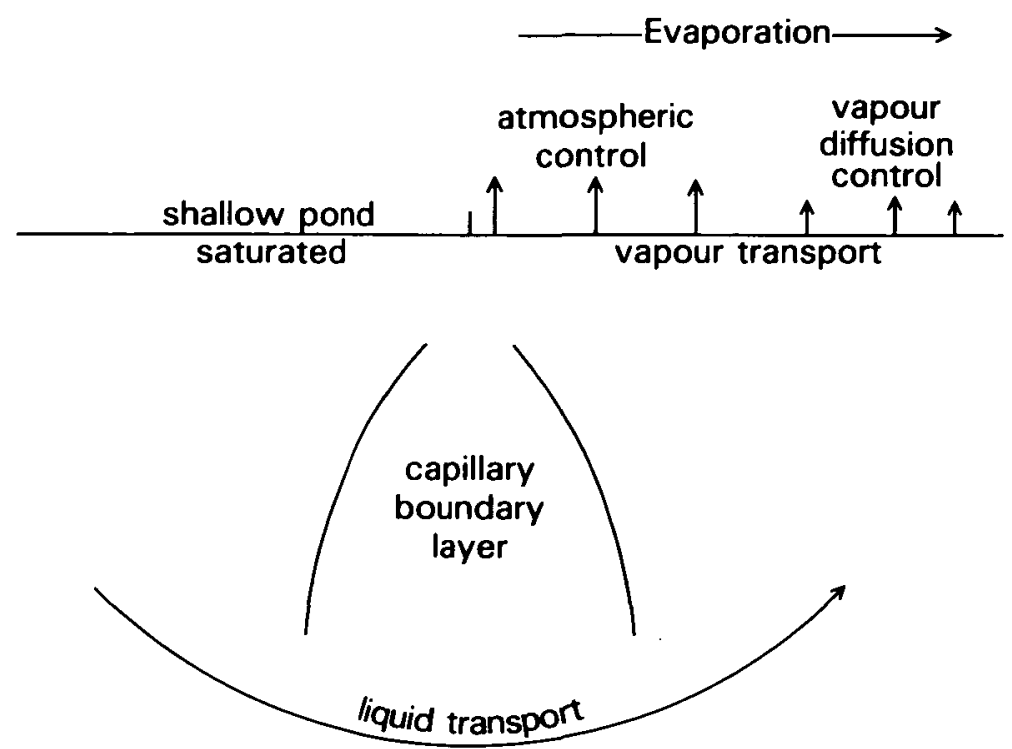

FIGURE 2. Liquid transport in a shallow pond.

an unsaturated porous medium occupied by water, the relative humidity $H$ satisfies

$$
H=\exp (g M \psi / R T),
$$

where $g$ is gravitational acceleration $\left(9.8 \mathrm{~m} / \mathrm{sec}^{2}\right), M$ the molecular weight of water $(0.018 \mathrm{~kg} / \mathrm{mole}), \psi$ water potential, $R$ the gas constant $(8.3$ joules/ ${ }^{\circ} \mathrm{K}$ mole) and $T$ is temperature $\left(300^{\circ} \mathrm{K}\right.$, say).

The quasilinear model of a porous medium used in this paper assumes that relative permeability and water potential are related exponentially,

$$
\kappa=\exp (\alpha \psi),
$$

and so (28) and (29) imply

$$
\begin{gathered}
H=\kappa^{e}, \\
\varepsilon=g M / \alpha R T .
\end{gathered}
$$

Field measurements and theoretical estimates of $\alpha$ suggest

$$
0.2 \mathrm{~m} \leq \alpha^{-1} \leq 5 \mathrm{~m}
$$

and so from (31),

$$
1.4 \times 10^{-5} \leq \varepsilon \leq 3.5 \times 10^{-4}
$$


In the vapour zone, relative humidity must be less than unity. Taking 0.99 as some typical value for $H$, and 0.00007 as an estimate for $\varepsilon$ gives $\kappa=\exp (-144)$. This infinitesimal value for $\kappa$ suggests the quasilinear model for liquid transport has broken down, and that the vapour zone forms not in response to the decrease in relative humidity in the soil, but from the decrease of liquid diffusivity, $D_{l}$, to a value approximately that for vapour diffusion, $D_{v}$ [5].

A conceptual model for evaporation about our pond is then saturated conditions under the pond; a constant evaporation rate due to atmospheric control to some distance from the pond, beyond which the evaporation rate decreases due to vapour control. In order to proceed analytically we simplify these boundary conditions by first replacing the saturated condition, (6), under the pond by

$$
2 \kappa^{*}-\frac{\partial \kappa^{*}}{\partial z}=2 ; \quad z=0, \quad x<0,
$$

which will be approximately true away from the pond's edge. To avoid confusion with conditions above we use the symbol $\kappa^{*}$. Secondly, we assume that approximate to the pond atmospheric conditions establish the evaporation rate at the maximum rate $E$ from saturated soil,

$$
\begin{gathered}
2 \kappa^{*}-\frac{\partial \kappa^{*}}{\partial z}=-2 E / F_{g} ; \quad z=0, \quad 0<x<x^{*}, \\
F_{g}=\rho g k_{1} / \mu,
\end{gathered}
$$

where $x^{*}$ is the nondimensional distance to which atmospheric control extends, and $F_{g}$ is the saturated conductivity, or equivalently the steady volumetric flux from gravity.

Thirdly, we assume that evaporation is zero beyond $x^{*}$,

$$
2 \kappa^{*}-\frac{\partial \kappa^{*}}{\partial z}=0 ; \quad z=0, \quad x>x^{*}
$$

The solution of (5) subject to (33)-(36) is then

$$
\begin{aligned}
\kappa^{*}(x, z) & =\frac{i}{\pi} \int_{-\infty}^{\infty} f(\xi) \exp \left[i \xi x+z\left(1-\sqrt{1+\xi^{2}}\right)\right] d \xi, \\
f(\xi) & =\left[F_{g}-E\left(e^{-i \xi x^{*}}-1\right)\right] / F_{g} \xi\left(1+\sqrt{1+\xi^{2}}\right)
\end{aligned}
$$


and so at the limiting position $x^{*}$ of atmospheric control on evaporation,

$$
\begin{aligned}
\kappa^{*}\left(x^{*}, 0\right)= & \frac{i\left(F_{g}+E\right)}{\pi F_{g}} \int_{-\infty}^{\infty} e^{i \xi x^{*}} d \xi / \xi\left(1+\sqrt{1+\xi^{2}}\right) \\
& -\frac{i E}{\pi F_{g}} \int_{-\infty}^{\infty} \frac{d \xi}{\xi\left(1+\sqrt{1+\xi^{2}}\right)}, \\
= & \frac{2\left(F_{g}+E\right)}{\pi F_{g}} \int_{1}^{\infty} \frac{e^{-\eta x^{*}} \sqrt{\eta^{2}-1} d \eta}{\eta^{3}}-\frac{E}{2 F_{g}} .
\end{aligned}
$$

The integral in (40) yields an exponentially damped function of $x^{*}$, and so for $x^{*}$ sufficiently great, $\kappa^{*}\left(x^{*}, 0\right)$ will become negative. Consequently we fix $x^{*}$ by requiring

$$
\kappa^{*}\left(x^{*}, 0\right)=0
$$

which from (40) yields

$$
\frac{e^{-x^{*}}}{\left(x^{*}\right)^{3 / 2}} \cong \sqrt{\frac{\pi}{2}} \frac{E}{2\left(E+F_{g}\right)},
$$

or

$$
x^{*} \cong \ln \left(1.6 F_{g} / E\right),
$$

since in nature $E$ is much less than $F_{g}$.

The total volumetric rate of loss of water from the soil, per unit length of pond perimeter, for the function $\kappa^{*}$ is $2 E x^{*} / \alpha$. Equating this to $T F_{e}$ gives from (43), (25), (35) and (10),

$$
\pi(\gamma-1) \theta^{\prime}(0) / 2 \cong\left(\pi E / F_{g}\right) \ln \left(1.6 F_{g} / E\right),
$$

and so $\gamma$ can be read off from Table 1 .

As an example, let $E$ be $0.005 \mathrm{~m} /$ day, and consider values of $F_{g}$ between 0.025 and $1 \mathrm{~m} /$ day. Then $\gamma$ varies between about 10 and 1.3 , and the corresponding nondimensional distances $x^{*}$ in (43) are respectively 2 and 6 . The corresponding additional total outflow is respectively between $63 \%$ and $5 \%$ greater than those for no evaporation.

These numerical examples indicate for freely draining soils (with $F_{g}$ equalling about $1 \mathrm{~m} /$ day) that evaporative flows are relatively unimportant compared to the capillarity-evaporative flows. However, for poorly draining soils, the converse may be true. This implies for poorly draining soils evaporative fluxes may be important, and flux-like equations derived subject to the assumption of zero evaporation may require alteration. 


\section{Conclusion}

The unsaturated steady flow from a shallow half-plane surface pond of water into a half-space of porous material has been discussed subject to the quasi-linear assumption that relative permeability is exponentially related to water potential, and that non-wetted surfaces suffer a volumetric flux loss proportional to the local value of relative permeability. The two latter assumptions were made in order to obtain linear equations, but whereas some experiments justify making the quasi-linear assumption, there appears to be no corresponding experimental support for the evaporative loss equation in (3).

The resulting two-dimensional linear mixed boundary value problem is solved by performing a Wiener-Hopf factorisation. A referee has pointed out that an essentially identical factorisation is described by Noble ([3], page 91) for electromagnetic diffraction from a half-plane. Consequently, the factorisation result is simply stated in this paper.

Inclusion of a linearised evaporative loss term in the unsaturated boundary region yielded an additional logarithmic expression. This logarithmic expression, absorbed into (18) featured prominently in the asymptotic results as well as in the evaluations of the total flow from the pond and the total evaporative flow from non-wetted regions.

\section{References}

[1] G. F. Carrier, M. Krook and C. E. Pearson, Functions of a complex variable (McGrawHill Book Co., New York, 1966).

[2] D. Hillel, Soil and water, physical principles and processes (Academic Press, New York, 1971).

[3] B. Noble, Methods based on the Wiener-Hopf technique, (Pergamon Press, London, 1958).

[4] J. R. Philip, "Steady infiltration from buried point sources and spherical cavities", Water Resour. Res. (4) (1968) 1039-1047.

[5] J. R. Philip, "Evaporation, and moisture and heat fields in the soil", J. Meteorol. 14 (1975) 354-366.

[6] K. Pruess, TOUGH Users Guide, Lawrence Berkeley Laboratory, report for the US Department of Energy, 1986.

[7] R. T. Waechter and J. R. Philip, "Steady two- and three-dimensional flows in unsaturated soil: the scattering analog", Water Resour. Res. (21) (1985) 1875-1887.

[8] G. J. Weir, "Steady infiltration from large shallow ponds", Water Resour. Res. (22) (1986) 1462-1468. 